\title{
La estrategia institucional de China hacia América Latina. Análisis comparado entre los foros Celac-China y Celac-Unión Europea
}

\author{
Mariano Mosquera* \\ Daniel Morales Ruvalcaba**
}

RESUMEN

En el 2008 China difundió el Libro blanco hacia América Latina con una estrategia de dos dimensiones: la promoción de la particularidad y el fomento de la omnidireccionalidad. Además, el Foro China-Celac (FCC), creado en el 2014, es la plataforma más importante de China para canalizar la cooperación hacia América Latina. Así, el presente estudio demuestra comparativamente que el FCC aplica las dos dimensiones de la estrategia china bajo tres principios institucionales: flexibilidad, no condicionalidad y multiplicidad.

Palabras clave: estrategia, instituciones, cooperación internacional, China, Celac
China's institutional strategy towards Latin American countries. A comparative analysis between Celac-China forum and Celac-European Union forum

\section{ABSTRACT}

In 2008 the People's Republic of China disseminated the White Paper to Latin American countries with a two-dimensional strategy:

\footnotetext{
* Mariano Mosquera. Doctor en ciencia política de la Universidad Nacional de Córdoba (Argentina). Research Fellow en el Centro de Estudios Lationamericanos de la Sun Yat-sen University (China) [mosquera@mail.sysu.edu.cn].

** Daniel Morales Ruvalcaba. Doctor en ciencias sociales por la Universidad de Guadalajara (México). Research Fellow en el Centro de Estudios Lationamericanos de la Sun Yat-sen University (China) y miembro del Sistema Nacional de Investigadores (México) [morales@mail.sysu.edu.cn].

Recibido: 22 de mayo de 2018 / Modificado: 19 de julio de 2018 / Aceptado: 23 de julio de 2018

Para citar este artículo:

Mosquera, M. y Morales Ruvalcaba, D. (2018). La estrategia institucional de China hacia América Latina. Análisis comparado entre los foros Celac-China y Celac-Unión Europea. OASIS, 28, pp. 123-149.
}

Dor: https://doi.org/10.18601/16577558.n28.08 
the promotion of particularity logic and the promotion of omnidirectionality. In addition, China-Celac Forum (CCF), created in 2014, is the most important platform for China to promote cooperation towards Latin American countries. Thus, the present study demonstrates comparatively that the CCF applies the two dimensions of the Chinese strategy under three institutional principles: flexibility, nonconditionality and multiplicity.

Key words: strategy, institutions, international cooperation, China, Celac

\section{INTRODUCCIÓN}

El presente trabajo tiene como objetivo principal identificar la estrategia institucional de China hacia los países de América Latina. La temática es por demás relevante tanto por el desarrollo de intercambios económicos y políticos entre América Latina y China en años recientes (Pastrana Buelvas y Vera Piñeros, 2017; Chaponniere y Salama, 2016; Yu, 2015; Choo, 2009; Cheng, 2006) como por el creciente interés académico por abordar la relación entre las partes (Armony y Pérez-Liñán, 2017; Zhang y Wang, 1988; Sidel, 1983). Además, en comparación con otras regiones del mundo, una gran cantidad de investigaciones de este tipo han puesto el foco en la calidad institucional de esta relación (Leiteritz y Coral, 2017; Song y Wagner-Brizzi, 2014).

Siguiendo el objetivo mencionado, nuestro objeto de análisis es la relación entre China y la región de América Latina en su conjunto, sin diferenciación por países. En este sentido, primero, describiremos patrones discursivos comunes de China hacia la región de América Latina (Hunston, 1996; Lyons, 1995). Luego, nuestro interés específico se centrará en describir la estrategia institucional, es decir, en las posibilidades de que el discurso chino hacia la región adopte características de prácticas regulares en el marco de espacios cooperativos (North, 1993; Olson 1993). De esta forma el objeto de investigación se acota a un espacio cooperativo que relaciona a China con América Latina y, luego, a un objeto comparado que contrasta con la relación entre la Unión Europea (UE) y América Latina a partir de un espacio cooperativo de características similares.

Esta investigación diferencia entonces entre la estrategia discursiva china -entendida como influencia de la retórica china (Corkin, 2014) - y su implementación como estrategia institucional -referenciada como una función institucionalista- observable a través de la creciente participación de China en organizaciones como la Organización de Naciones Unidas (ONU) o la Organización Mundial de Comercio (OMC). Sin embargo, en los mencionados casos de la ONu y de la OMC se trata de una participación de China en marcos preestablecidos (Urdinez y Masiero, 2015; Prime, 2006; Braun, 1973), a diferencia del caso de estudio de la Comunidad de Estados Latinoamericanos y Caribeños (Celac) donde consideramos la posibilidad de una mayor influencia de China en la forma de rule-maker (Gao, 2007).

Para el caso de la estrategia discursiva china hemos tomado como base el Documento 
sobre la Política de China hacia América Latina y el Caribe ${ }^{1}$, de noviembre del 2008. Este documento conocido como el Libro blanco (LB) de China hacia América Latina define la estrategia china de forma explícita, en un sentido discursivo. Se trata del primer documento de China de este tipo, exclusivo para la región de América Latina ${ }^{2}$. Además, el LB 2008 es una referencia que se replica de forma recurrente en la retórica diplomática de China hacia la región (Abdenur y De Souza Neto, 2013). En él se afirma que:

La elaboración por parte del Gobierno chino del Documento sobre la Política hacia América Latina y el Caribe tiene como propósito manifestar con mayor claridad los objetivos de la política china hacia la región, plantear los principios rectores de la cooperación en las diversas áreas durante un determinado período del futuro y promover el continuo desarrollo sano, estable e integral de las relaciones sino-latinoamericanas y sino-caribeñas.

Por otra parte, en cuanto a la estrategia institucional, el Foro China-Celac (FCC) posee un diseño y un desarrollo institucional (Tzili, 2017) que permite considerarlo representativo para un estudio de este tipo. El FCC es un espacio cooperativo entre China y América
Latina donde consideramos que es posible identificar una explícita estrategia institucional de China hacia la región. La Celac incluye a los treinta y tres países de la región y fue creada en diciembre de 2011, lo cual hace relevante su representación y, a la vez, torna muy factible la influencia china sobre su reciente institucionalidad en el marco del foro.

De esta forma, nuestra hipótesis de trabajo es que el FCC aplica institucionalmente la estrategia del discurso del LB 2008 en sus dos dimensiones -fomento de la particularidad y promoción de la omnidireccionalidad-, mientras que la Celac no aplica estos patrones estratégicos con otras regiones.

Las dos dimensiones mencionadas en nuestra hipótesis serán desarrolladas en el marco conceptual de este trabajo. De todas formas, anticipamos que el fomento de la particularidad se relaciona con el énfasis del discurso chino por encontrar características particulares en cada relación entre países y evitar así el condicionamiento externo con recetas uniformes (Bolinaga y Slipak, 2015; Huang, 2010). Por su parte, la omnidireccionalidad se relaciona teóricamente con el punto anterior, es decir, se trata de promover múltiples direcciones temáticas de cooperación, aunque no necesariamente de forma específica en su contenido.

\footnotetext{
1 Documento sobre la Política de China hacia América Latina y el Caribe, del gobierno de la República Popular China, con fecha 5 de noviembre de 2008.

2 Esta investigación analiza fundamentalmente el Libro blanco de noviembre de 2008 y no la segunda versión del 2016 por diversas razones. Es el primer documento que define explícitamente bases y esto nos permite estudiarlo como antecedente-marco de instrumentos posteriores en materia de cooperación China-América Latina. También, nos brinda un mayor recorrido temporal para relevar análisis académicos sobre su retórica e implementación. Finalmente, el análisis comparado entre los dos libros blancos no arrojó significativas diferencias (en frecuencia léxica de términos clave es de 3,8\%) en las dimensiones de interés de esta investigación.
} 
Para comprobar nuestra hipótesis, contrastaremos el FCC con otro mecanismo de diálogo similar que tiene América Latina: el Foro Celac-Unión Europea. Este procedimiento comparativo obedece a dos premisas metodológicas:

1. El FCC es la plataforma institucional más relevante que tiene China para canalizar su relación hacia América Latina.

2. La Celac ha establecido relaciones institucionales con la UE, permitiendo un análisis comparado entre el fCC y el Foro Celac-Unión Europea.

El presente trabajo plantea un vacío de conocimiento como problema ${ }^{3}$, y lo resuelve con un análisis descriptivo y comparado de un caso representativo, guiado por el objetivo general y por la hipótesis de trabajo. Como conclusión describiremos los principios institucionales de la estrategia china hacia América Latina que son exclusivos de esta relación.

El primer apartado de esta investigación describe antecedentes de estudio sobre las relaciones entre China y América Latina. En particular, se señala la importancia de China como la mayor potencia emergente de las últimas décadas y se destaca el debate sobre la especificidad de su sistema político-económico y de vinculación exterior. Además, se rescatan antecedentes sobre la Celac y el FCC. Luego, se prueban las dos premisas metodológicas de nuestro objeto comparado de estudio antes enumeradas. La prueba empírica (cuantitativa) sobre la relevancia comparada de los organismos de integración regional para China es un test original de este trabajo.

En un segundo apartado, se definen conceptos, especialmente, el de estrategia y sus dimensiones discursivas y principios institucionales. Las definiciones conceptuales, en general, son inductivas desde los propios documentos analizados lo que permite identificar las variables más operativas (categorías) para la prueba empírica de la hipótesis de trabajo.

El tercer apartado es de tipo metodológico (Badía, 1973). Aquí se articula el análisis normativo, el método comparado, el análisis por frecuencia léxica y el análisis temáticoinstitucional. La cuarta sección ofrece resultados comparados, tanto cuantitativos como cualitativos.

Finalmente, se presentan las conclusiones y un espacio de discusión que busca articular los hallazgos de la investigación con referencias generales sobre la estrategia institucional de China hacia América Latina, con especial atención sobre las implicancias para futuras agendas de investigación.

\section{ESTADO DEL ARTE DE LAS RELACIONES CHINA - AMÉRICA LATINA}

Los estudios sobre la relación entre China y América Latina son abundantes. Entre ellos, queremos destacar: desde el ámbito histórico, los trabajos de Frank Mora (1997, 1999),

\footnotetext{
En este sentido la pregunta de investigación ha sido: ¿Cuál es la estrategia institucional actual de China hacia América Latina?
} 
quien ha hecho una revisión de las relaciones sino-latinoamericanas desde la década de los 70 hasta finales del siglo xx; desde lo económico, sobresalen las investigaciones de Rhys Jenkins (2012), Liska Gálvez (2012), Barbara Kotschwar (2014), Jean-Rafhaël Chaponnière y Pierre Salama (2016) quienes, a grandes rasgos, han identificado las principales fortalezas y desafíos de esta relación, en especial en lo concerniente al comercio; y desde lo político, las publicaciones de Jiang Shixue (2006), Joseph Cheng (2006), Jaewoo Choo (2009), Evan Ellis (2009), Benjamin Creutzfeldt (2013) y Lei Yu (2015), quienes han dado seguimiento al creciente entendimiento entre China y Latinoamérica. Por lo tanto, se trata, en la mayoría de los casos, de estudios geoeconómicos y geopolíticos que destacan la función actual de la vinculación entre China y los países de América Latina (Haro Navejas y Hernández Mendoza, 2016; Cornejo, 2013; Gálvez, 2012; Jaramillo, Lehmann y Moreno, 2009; Ellis, 2009).

La posibilidad real y latente de que potencias emergentes de la actualidad puedan disputar la hegemonía estadounidense en el siglo XxI, ha despertado mucho interés y especulación. Con respecto a ello, China ha sido el país más destacado en lo que refiere al incremento de poder nacional (Morales Ruvalcaba, 2015). A pesar de la mejoría en su posicionamiento estructural, China sigue siendo un país en desarrollo (Morales Ruvalcaba, 2013; Wallerstein, 2003a, 2003b; Terlouw, 2002; Arrighi y Drangel, 1986), pero con proclividad a convertirse en potencia hegemónica (Morales Ruvalcaba, 2017; Ikenberry, 2008; Rosecran- ce, 1987; Modelski, 1978). Para consolidar su presencia internacional, China necesita estabilizar su poder económico-militar, incrementar significativamente su poder económico-social y afianzar su poder comunicativo-cultural (Rocha Valencia y Morales Ruvalcaba, 2018). Como bien señala Andrés Serbín, en este proceso parece encontrarse Beijing, asociando la proclividad hegemónica china con "acuerdos como el Regional Comprehensive Economic Partnership, la nueva ruta de la seda (One Belt, One Road en su conexión con Asia Central y Europa y en su proyección marítima), el Asian Infrastructure Investment Bank y el Free-Trade Area of the Asia-Pacific, entre otros instrumentos" (Serbin, 2017, p. 78). Como parte de dicho despliegue geoeconómico y geopolítico América Latina ocuparía un lugar estratégico.

En este contexto internacional, uno de los debates más relevantes es el que gira en torno a la calidad institucional de esta relación entre China y América Latina, ya que de la institucionalidad o de su falta dependen las condiciones de sustentabilidad económica en América Latina o de orientación hegemónica china (Haynes, 2017; Escudé, 2012; Beeson, 2009; Arrighi, 2007), entre otros efectos posibles. En este sentido, el estudio de la estrategia china desde su discurso o retórica es marginal, con escasas menciones para contextualizar o dar apoyo a argumentos que son estudiados, en general, desde dimensiones materiales. Tampoco son recurrentes los estudios de casos para analizar en profundidad las implicancias institucionales de la relación entre China y América Latina. Pueden mencionarse como relevantes los de Ray, Gallagher, López y Sanborn (2015). 
Por otra parte, es relevante destacar que el sistema político y económico de China ha sido ampliamente estudiado por su particularidad. Muchas investigaciones se han centrado en la especificidad de las políticas o medidas internas y en el impacto de estas en el crecimiento económico de China desde la reforma y apertura de diciembre de 1978 (Wang, 1988; Garner, 2008; Chow, 2010). Esta especificidad también se ha extendido a la política exterior de China. Los estudios de Liu (2017) y Su (2017) refieren a los elementos de particularidad en la vinculación de China con el mundo. En el marco de nuestro trabajo importa comprender que el caso de China ha dado lugar a múltiples debates -desde distintas disciplinas y enfoques- sobre su especificidad y cómo esto alcanza a su diplomacia, incluyendo su relación institucional con los organismos de integración regional.

En lo que respecta a la especificidad de nuestro caso de estudio, destacamos que la Celac es un organismo regional de reciente constitución - diciembre de 2011-que agrupa a la totalidad de los treinta y tres Estados de la región y que ha nacido con una vocación geopolítica manifiesta, que posiciona a esta comunidad en la cúspide de los procesos de integración regional de América Latina (Rocha Valencia, 2014). De ahí el posible interés de China por el establecimiento de vínculos destacados con este organismo (Ayuso y Villar, 2016). Numerosos estudios pueden mencionarse sobre la Celac, a pesar de su creación reciente. Los trabajos de
Romero (2014), Bonilla Soria (2014) y Segovia (2013), resaltan su función.

Por su parte, el denominado FCC fue constituido el 17 de julio del 2014. China, en sus documentos oficiales, lo describe como una plataforma estratégica de cooperación. La relevancia para nuestro estudio se debe a que se trata de una iniciativa de cooperación con participación china y con un claro desarrollo de instituciones para la cooperación (Tzili, 2017). Es decir, el FCC posee disposiciones institucionales y reglas de funcionamiento y un Plan de Cooperación (2015-2019) bien definido, entre otras claras prácticas institucionales. En este sentido, los estudios sobre la relación Celac-China, como los de López, Rodil, Martínez y Valdez (2015), se centran en el comercio y en la especialización productiva. Otros estudios sobre el FCC, se focalizan en la misma materia, como Rosales (2015). Sin embargo, son menos comunes los estudios de tipo institucional.

Respecto a la representatividad metodológica del FCC para nuestro trabajo recordamos la primera premisa mencionada con anterioridad:

\section{EI FCC es la plataforma institucional más relevante de China para canalizar su relación hacia América Latina ${ }^{4}$}

Para confirmar esto, en esta investigación se construyó un corpus (McEnery y Wilson, 2001) y se realizó un análisis de contenido

$4 \quad$ El Libro blanco del 2016 dice: "China propulsará su cooperación en conjunto con América Latina, con el Foro China-Celac como plataforma principal, ateniéndose, en la cooperación, al principio de trato de igualdad, el objetivo de beneficio mutuo y ganancia compartida, las formas flexibles y pragmáticas y el espíritu de apertura e inclusión”. 
(Kolbe y Burnett, 1991) de la agenda diplomática de China hacia América Latina, tomando como unidad de contexto las comunicaciones del Ministerio de Relaciones Exteriores de la República Popular China entre diciembre del 2008 y diciembre del 2016. Las comunicaciones incluyen toda la actividad diplomática china hacia América Latina. El recorte temporal se estableció tomando el período entre el primer y el segundo LB de China hacia América Latina. El corpus relevado posee comunicaciones en forma de entrevistas, discursos políticos, notas en medios periodísticos, descripción de encuentros entre funcionarios y declaraciones conjuntas, entre otros formatos narrativos. Se trata de un corpus de 507 comunicaciones y de 236.798 tokens finales. Por otra parte, se construyó un gazetteer con los términos - unidad de registro-que representan a los distintos organismos de integración regional de América Latina. Cabe destacar que se realizaron operaciones morfológicas con dichos términos, para garantizar una búsqueda exhaustiva de información. Los organismos relevados fueron:

AP: $\quad$ Alianza del Pacífico.

Caricom: Caribbean Community o Comunidad del Caribe.

Sica: Sistema de Integración Centroamericana.
CAN: $\quad$ Comunidad Andina.

Alba: Alianza Bolivariana para los Pueblos de Nuestra América.

Mercosur: Mercado Común del Sur.

Unasur: Unión de Naciones Suramericanas.

Celac: Comunidad de Estados Latinoamericanos y Caribeños.

Se utilizó el software libre GATE (General Architecture for Text Engineering) de la Universidad de Sheffield para el relevamiento de términos por medio de la construcción del gazetteer. Tanto el corpus ${ }^{5}$ como el gazetteer ${ }^{6}$ pueden encontrarse en repositorios de datos dataverse de la Universidad de Harvard, para la replicación del presente estudio. Como resultado, podemos afirmar que la agenda diplomática de China hacia los organismos de integración regional se caracteriza por una gran disparidad, en cuanto al volumen de actividad, entre la Celac y el resto de las organizaciones, tanto a nivel de comunicaciones (documentos) como a nivel de tokens. Por su parte, organizaciones como Sica, CAN y Alba no tienen menciones en la actividad diplomática china.

Además, el punto 7 de la Parte IV del Libro blanco del 2016 está destinado exclusivamente a resaltar la función institucional del FCC como instancia principal de la cooperación entre China y América Latina, destacando el principio de no-condicionalidad y la cooperación en múltiples áreas en el marco del foro.

5 Mosquera, M.; Morales Ruvalcaba, D. (2017). Corpus de Comunicaciones Diplomáticas de China hacia América Latina, doi: 10.7910/DVN/KYOWIS, HarvardDataverse, V1.

6 Mosquera, M.; Morales Ruvalcaba, D. (2017). Gazetteer de Organizaciones de Integración Regional de América Latina, doi: 10.7910/DVN/CZGMMS, HarvardDataverse, V1. 
Gráfico 1

Cantidad de menciones de los distintos organismos de integración regional en la agenda diplomática china hacia América Latina.

Por documentos y tokens entre 2008 y 2016

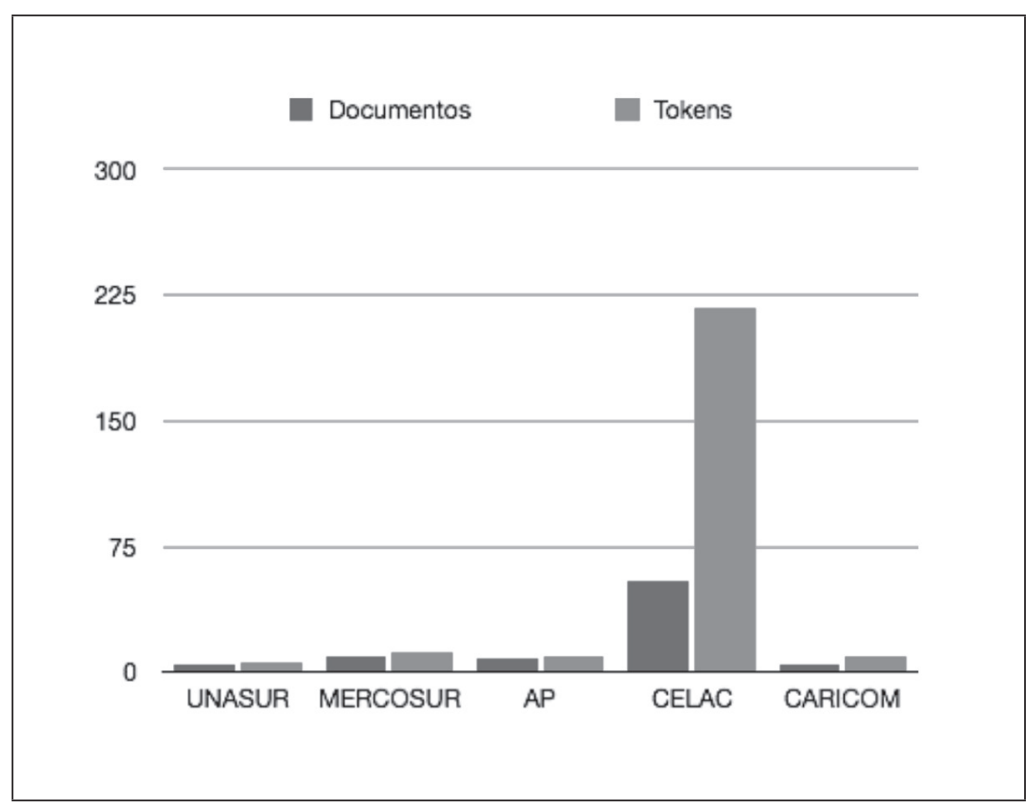

Fuente: elaboración propia con base en comunicaciones del Ministerio de Relaciones Exteriores de la República Popular China, 2008 a 2016.

La actividad con la Celac comienza en diciembre del 2011, con una comunicación china a los presidentes de Chile y de Venezuela felicitándolos por su constitución. La actividad diplomática de China con la Celac tiene su punto máximo en 2015 con la realización en territorio chino del FCC. 


\section{Gráfico 2}

Menciones a Celac en la agenda diplomática china hacia América Latina. Por año y según documentos y tokens

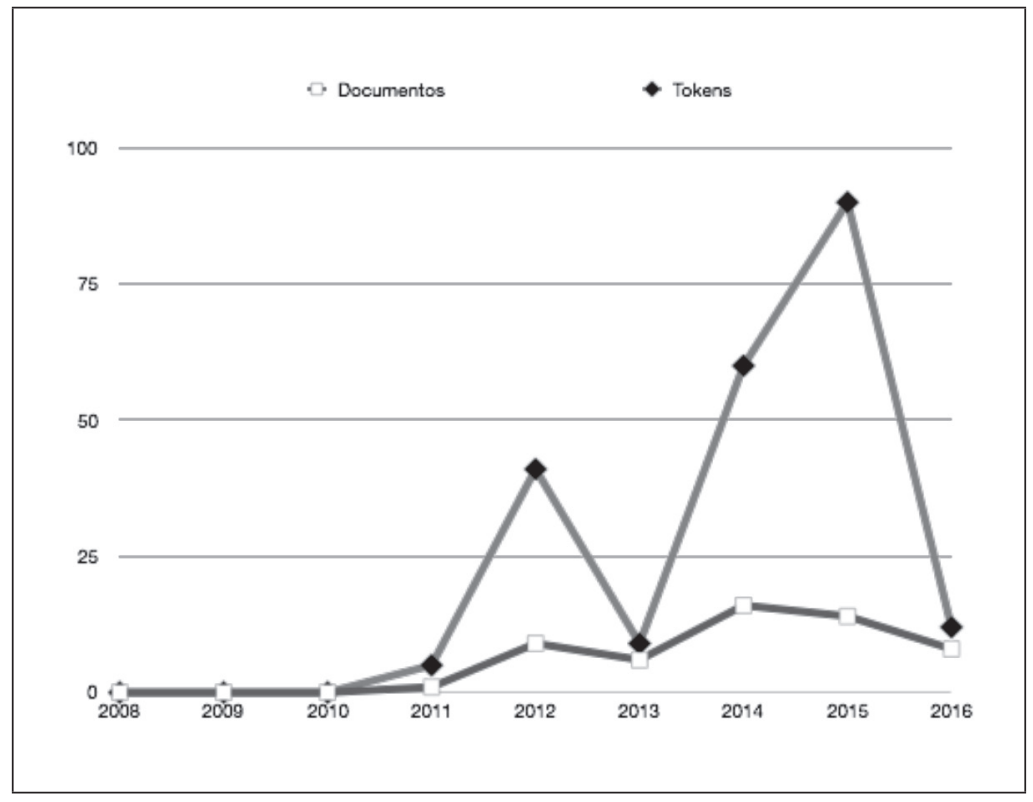

Fuente: elaboración propia con base en comunicaciones del Ministerio de Relaciones Exteriores de la República Popular de China, 2008 a 2016.

Suponemos, en este punto del argumento, la existencia de mecanismos de selección de la Celac por parte de China como espacio organizacional para traducir su estrategia discursiva en prácticas institucionales. Esto puede explicarse desde el punto de vista de la identificación oportuna de una comunidad de prácticas transnacionales con condiciones de representatividad (Adler, 2005). Este es el caso de la participación de los treinta y tres Estados de América Latina y el Caribe en la Celac. Además, se suma la identificación por parte de China de la reciente construcción institucional de la Celac (de diciembre de 2011) que puede permitir condiciones para influir en forma de rule-maker (Gao, 2007).

Por otra parte, respecto a la segunda premisa metodológica de nuestro objeto de estudio, recordamos su formulación:

\section{La Celac ha establecido relaciones institucionales con la UE, permitiendo un análisis comparado entre el FCC y el Foro Celac-Unión Europea}

La relación Celac-UE se refleja en distintos documentos, como la Declaración de Santiago de enero de 2013, la Declaración de Bruselas 
de junio de 2015 y la Declaración de Santo Domingo de octubre de 2016. Además, la relación posee un Plan de Acción conjunta que data de 2015. Algunos antecedentes académicos destacados que han trabajado esta relación con distintos enfoques son los estudios de Ríos Sierra (2014), Casanueva (2013), De Onis (2013) y Walker y Casanueva (2012). Estos trabajos se centran nuevamente en la función geoeconómica y geopolítica de la relación, sin profundizar en los principios o diseños institucionales.

En definitiva, la relación entre China y América Latina ha sido estudiada de manera recurrente, incluso destacando que son importantes las variables institucionales (Tzili, 2017). Sin embargo, estudios de caso representativos, estratégicos-institucionales y de tipo comparativo, son aún escasos.

\section{LA ESTRATEGIA DISCURSIVA E INSTITUCIONAL CHINA HACIA AMÉRICA LATINA}

Dado que las unidades de análisis seleccionadas en esta investigación refieren a prácticas discursivas (Ruggie, 1998) y que de allí se pueden inferir funciones para analizar, en definitiva, relaciones de poder y legitimación (Finnemore y Sikkink, 2001), es que esta investigación aproxima teóricamente en lo que se conoce como constructivismo en relaciones internacionales (Vitelli, 2014; Wendt, 1999; Hopf, 1998). Lo que buscamos proponer al lector son los puntos de encuentro de esta perspectiva teórica - desde la construcción del objeto de estudio- en su relación con la estructura del sistema internacional; es decir, con nuestro análisis buscamos una mejor comprensión de la articulación entre agente (Estados) y estructura.

Luego, para pasar del enfoque teórico a un nivel de marco conceptual, trabajaremos los siguientes aspectos fundamentales: la definición de estrategia, la identificación de las dos dimensiones estratégicas del discurso chino, la relación con los principios institucionales y su operativización en las categorías del análisis empírico.

El concepto de estrategia se define, en nuestro trabajo, de la siguiente forma combinada:

1. En un sentido político del término, con énfasis en la habilidad de guiar (Giddens, 2015; Porter, 2011).

2. En un sentido relacional del término, con referencia a la interacción entre el medio interno y el entorno (Hoffer y Schendel, 1978).

Varias cuestiones pueden destacarse aquí con respecto a esta definición de estrategia, que representa un concepto principal para nuestra investigación. En relación con el punto uno: la mención recurrente en el discurso chino a la necesidad de liderazgos políticos para poder desarrollar con éxito la cooperación. Respecto al punto dos: la toma de decisiones según el contexto como una cuestión también central en el discurso político chino (Xi, 2014). Además, obsérvese que no hay ninguna referencia en esta definición seleccionada sobre estrategia, a los medios y a los fines o a su interrelación.

La estrategia china hacia América Latina es definida en el LB del 2008 con base en sus dos dimensiones propias: la promoción de la particularidad y el fomento de la omnidireccionalidad. 
Tal como veremos a continuación, ambas dimensiones están en íntima relación con nuestro tipo de definición combinada de estrategia.

La primera dimensión del LB refiere a la valorización que otorga la estrategia china al hecho de encontrar de forma dinámica, en cada relación particular, las propias condiciones de la cooperación. Es decir, definir características propias, en cuanto al contenido de la cooperación, en contextos particulares y, por lo tanto, evitar generalizaciones o recetas comunes para todos los países (Li, 2014). Se trata de una dimensión política que se articula con el punto dos de nuestra definición combinada de estrategia, ya que lo que requiere es establecer los límites entre el medio interno y el entorno ${ }^{7}$. En este sentido el LB nos plantea: "Las naciones latinoamericanas y caribeñas exploran activamente los caminos de desarrollo ajustados a sus realidades nacionales". Esta dimensión se relaciona con el denominado Consenso de Beijing (Fanjul, 2011; Cooper Ramo, 2004), e incluso, con la política doméstica china y su énfasis en las "características chinas" del desarrollo (Xi, 2014; Herrera-Feligreras, 2017).

La segunda dimensión del LB 2008 trata de abarcar una gran cantidad de áreas y temas de cooperación. Desde un punto de vista teórico, esto se debe a que se supone que una lógica particular requiere múltiples escenarios de intervención en lugar de pocos espacios uniformes. También se supone que las múltiples direcciones no deben ser del todo específicas, para no condicionar la construcción de las ca- racterísticas particulares. La parte IV del LB, denominada "Fortalecimiento de la Cooperación Omnidireccional entre China y América Latina y el Caribe", se dedica exclusivamente a esta dimensión, definiendo las siguientes cuatro áreas: política; económica; cultural y social; y de paz, seguridad y justicia. Cada una de estas áreas se relaciona con múltiples temáticas: seis en el caso del área política; catorce para el área económica; once para el área cultural y social; y tres para el área de la paz, la seguridad y la justicia.

Hasta ahora estas han sido referencias discursivas de la estrategia china (Lyons, 1995). Sin embargo, una aplicación del tipo institucional como prácticas regulares (North, 1993) requiere tener presente que las instituciones de cooperación regional no condicionen las particularidades nacionales. En este sentido, consideramos que deben tenerse en cuenta tres ejes de implementación institucional:

- Flexibilidad institucional: se trata de una dinámica de adaptación permanente de los acuerdos, objetivos y temas. Se refleja en procesos institucionales de debate y diálogo constante, pero bajo condiciones de liderazgo político que permitan decidir sobre el cambio institucional.

- No condicionalidad: se refiere a un rechazo de los acuerdos como bases rígidas, que son externos y están por sobre los actores de la cooperación. Se visibiliza en condiciones institucionales de vigilancia constante de la autonomía.

\footnotetext{
$7 \quad$ Si bien excede esta investigación, es importante aclarar que esta dimensión tiene relación con principios confucianos y pragmáticos en la cultura política china. Puede verse esta vinculación en Gómez Díaz (2017) y Li y Wu (2016).
} 
- Multiplicidad: se define como una promoción de la diversidad, acorde con los principios anteriores. Se pone en evidencia en la diversificación de áreas institucionales por sobre las tareas de coordinación institucional.

Estos tres principios institucionales son explícitamente referenciados en la Declaración de Beijing del FCC (DBe), aunque su definición tal como ha sido expuesta se trata de una construcción indirecta, dado el sentido en la declaración referida. Además, destacamos que el principio de flexibilidad puede vincularse a una conceptualización más organizacional como en Bourgeois (1980) y el principio de no condicionalidad con características autonómicas de la política exterior como en Simonoff (2009), Tokatlian y Carvajal (1995), y Jaguaribe (1973).

Los principios institucionales dan lugar a las cinco categorías comparativas para la prueba empírica de nuestra hipótesis de trabajo ${ }^{8}$. Se trata de la mención en los documentos institucionales de: procesos de diálogo (1), consensos estables (2), objetivos (3), bases preestablecidas (4) y temáticas (5).

Dada nuestra hipótesis de investigación se espera que el FCC adopte cierta forma de flexibilidad institucional y de no condicionalidad en estas cinco categorías, y que considere a la omnidireccionalidad a partir de la constitución de múltiples áreas y temas de cooperación institucional, aunque permitiendo la adaptación de dichas temáticas. De cumplirse nuestra hipótesis, que vincula estas dimensiones con la estrategia de China, la relación institucional Celac-UE no debería presentar el mismo patrón.

Nos parece importante realizar aquí una aclaración sobre el concepto de cooperación. Hemos preferido utilizar este término atendiendo a que es parte de la retórica china, y simplemente como concepto por definir en el marco de relaciones entre países o regiones. Es decir, este trabajo supone que la propia prueba empírica puede orientar de distintas formas la definición de cooperación. La forma como China entiende la cooperación, con base en su estrategia, es distinta a como la define la UE. La relación de ambos con la Celac así espera demostrarlo.

Finalmente, como conclusión de esta sección, nos interesa mencionar que el LB 2008 en su parte V, "Relaciones entre China y las Organizaciones Regionales Latinoamericanas y Caribeñas", específicamente, afirma:

El Gobierno chino aprecia el papel relevante que desempeñan las organizaciones regionales y subregionales de América Latina y el Caribe en la salvaguardia de la paz y la estabilidad regionales y la promoción de la unidad y el desarrollo, así como la integración de la región y las apoya a que pongan en juego su positiva influencia en los asuntos regionales e internacionales. La parte china continuará fortaleciendo el intercambio, la consulta y la colaboración con las organizaciones pertinentes en los diversos terrenos.

8 De esta forma los parámetros de análisis siguen una lógica escalonada de operativización: dimensiones estratégicas discursivas, principios institucionales y categorías de análisis empírico. 
De esta forma, se destaca un apartado especial del LB 2008 en relación con las organizaciones regionales. Esta mención, repite las fórmulas de mantenimiento de la paz y la estabilidad para la unidad y el desarrollo, que son comunes en la retórica china. Sin embargo, es importante resaltar que China les asigna a este tipo de organizaciones regionales una función política de influencia en los asuntos internacionales. Ocho años después, el Libro blanco del 2016 menciona al FCC como resultado institucional de la cooperación iniciada con el primer LB: "El establecimiento del Foro entre China y la Comunidad de Estados Latinoamericanos y Caribeños (Foro ChinaCelac) ha brindado una nueva plataforma para la cooperación China-América Latina”.

\section{COMPARATIVA DE LOS FOROS CELAS- CHINA Y CELAC-UNIÓN EUROPEA}

Si bien hemos establecido que nuestro análisis se aproxima teóricamente al constructivismo, también es importante señalar -siguiendo a Vitelli- que para nosotros "no existe una metodología constructivista, sino que los argumentos constructivistas son compatibles con la utilización de los más variados métodos de investigación actualmente en uso en las ciencias sociales" (Vitelli, 2014, p. 139). En este sentido, la metodología aquí empleada, en un primer nivel, trata de un análisis normativo (Hadfield, 2005; Finnemore, 1996), debido a que se utilizan documentos oficiales para la identificación de la estrategia discursiva e institucional de China hacia América Latina. Específicamente nos referimos a:
- Documento sobre la Política de China hacia América Latina y el Caribe, de noviembre del 2008.

- Declaración de Beijing del FCC, de enero del 2015.

- Declaración de Bruselas de Celac-UE, de junio del 2015.

- Plan de Cooperación (2015-2019) del FCC, de enero del 2015.

- Plan de cooperación de Celac-UE, de junio del 2015.

Luego, para un adecuado análisis de la estrategia china se recurre al método comparado (Lijphart, 1971). Es decir, a la identificación de similitudes y diferencias (Peters, 1998; Ragin, 1987) entre las referencias documentales. En este contexto el LB 2008 aparece como nexo de la comparación, al tratarse de la referencia estratégica discursiva.

Así, en la comparación para la identificación de la lógica de la particularidad utilizamos la Declaración de Beijing y la Declaración de Bruselas, por tratarse de documentos políticos del mismo año. Actuando como nexo discursivo de esta comparación solo remitimos a los apartados políticos del LB - prólogo, apartado I, apartado II y apartado III-. Utilizamos un análisis de frecuencia léxica (Sinclair, 1991), vinculado con la identificación del sentido de los términos en su propio contexto (Rodríguez Sabiote, Lorenzo Quiles y Herrera Torres, 2005), lo que permite representar los términos clave y sus adaptaciones morfológicas de forma cuantitativa.

Para la comparación de la promoción de la omnidireccionalidad, empleamos el Plan de Cooperación del FCC (2015-2019) y el Plan de 
cooperación de Celac-UE del 2015. Del LB en su rol de nexo discursivo de la comparación, solo se tiene en cuenta su parte IV, "Fortalecimiento de la Cooperación Omnidireccional entre China y América Latina y el Caribe". En este caso se trata de un análisis de tipo temático-institucional (Giddens, 2015; Greif y Laitin, 2004; North 1993), identificando los espacios institucionales establecidos con base en ciertos límites -específicamente, de los temas y de sus relaciones-. Se buscan, también, referencias con respecto a la posibilidad de adaptar o no estas temáticas.

Por último, es necesario resaltar que nuestro trabajo se presenta como un estudio de caso (Breault y Callejo Pérez, 2012; George y Bennet, 2005) que intenta posibilitar inferencias para la generalización en torno a la estrategia institucional de China hacia América Latina.

\section{DIMENSIONES Y PRINCIPIOS DE LA ESTRATEGIA INSTITUCIONAL CHINA}

A continuación presentamos dos apartados -con sus correspondientes pruebas empíricassobre cada una de las dimensiones de nuestra hipótesis de trabajo.

\section{a. Particularidad}

Para la comprobación de la estrategia de fomento de la particularidad, realizamos un gráfico comparado de nube de palabras. Se trata de términos clave, de alta frecuencia léxica, en la Declaración de Beijing (DBe) y en la Declaración de Bruselas $(\mathrm{DBr})$.

\section{Gráfico 3}

Nube de palabras comparada.

Declaración de Beijing (izquierda) y Declaración de Bruselas (derecha)

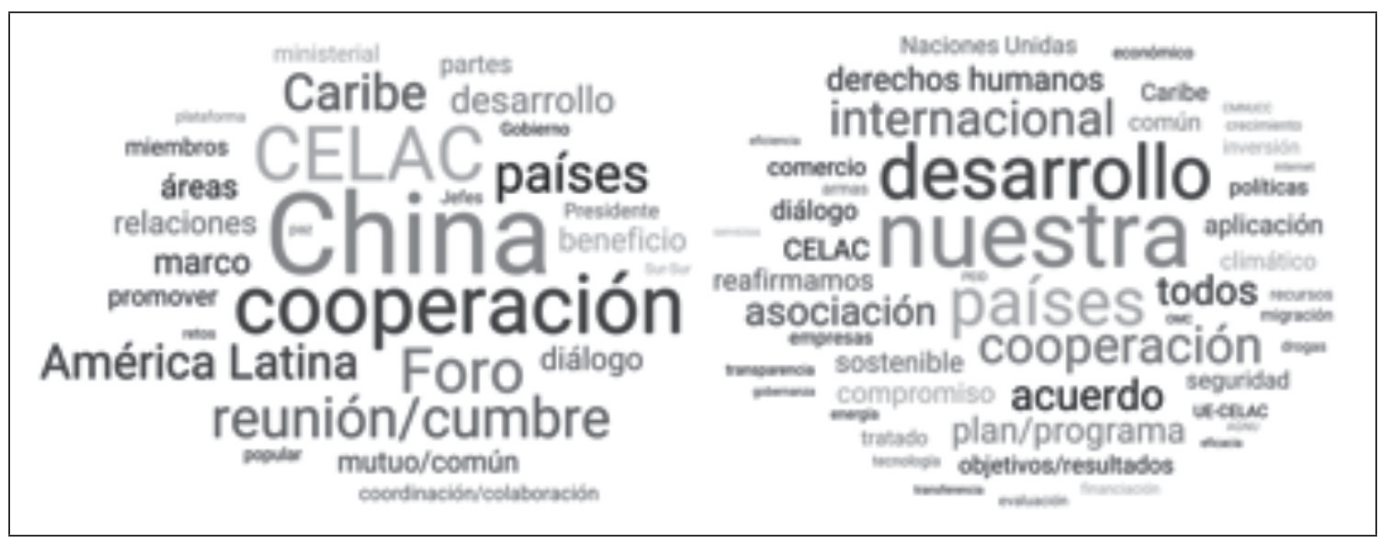

Fuente: elaboración propia con base en la Declaración de Beijing (2015) y la Declaración de Bruselas (2015). 
A partir del Gráfico precedente, describimos comparativamente las cinco categorías definidas para la prueba empírica: procesos (1), consensos (2), objetivos (3), bases (4) y temáticas (5). Esta comparación se basa en los detectados términos clave y de alta frecuencia léxica.

En cuanto al proceso institucional, tanto el LB como la DBe y la DBr buscan definir la noción de cooperación. De ahí que la mención de este término y los sinónimos de sentido utilizados sea alta en los tres casos. En el LB, la referencia a una construcción "mutua" y "compartida" de las relaciones cooperativas tiene una alta frecuencia. Lo mismo sucede en la DBe. Otros términos como "diálogo", "reunión", "plataforma" o "colaboración" son repetidos en el mismo sentido tanto en el LB como en la DBe. Incluso, en algunos apartados de estos documentos se explicita que el "diálogo" es el fin de la propia cooperación. De esta forma, la caracterización del proceso institucional tiene una alta frecuencia en el LB y en la DBe, aunque no sucede igual en el caso de la DBr.

A diferencia del LB y la DBe, la DBr prefiere centrarse en los consensos estables. Las referencias a alcanzar "acuerdos" o a adoptar "compromisos" son importantes en frecuencia de aparición, situación que no se aprecia en la DBe. En la DBr, estos consensos y su estabilidad a largo plazo se relacionan con el término "sostenible", también de alta frecuencia.

A nivel de objetivos -la tercera categoría de nuestra prueba sobre los documentos normativos-, el LB plantea el "desarrollo" y la "paz". Igual situación evidencia la DBe, mientras que la $\mathrm{DBr}$ se centra en el desarrollo y ofrece una referencia marginal a la paz. A la vez, es necesario destacar que la mención del desarrollo no es siempre una referencia a objetivos en el LB y en la DBe, sino que en numerosas oportunidades se refiere a un punto de partida entre China y América Latina por ser ambos actores en "vías de desarrollo". Por esta misma razón, para el agregado de frecuencias en la categoría objetivos, solo se incluye al "desarrollo" cuando se vincula con metas, tarea que requirió de un proceso de desambiguación (Sinclair, 1991). La DBe también prefiere centrarse en "retos" más que en objetivos, mientras que la DBr llega incluso a proponer metodologías de planificación muy específicas para la identificación y evaluación de las metas y sus resultados.

Por otra parte, tanto el LB como la DBe "promueven", más que nada, una nueva relación. En cambio, la DBr "reafirma” o "aplica" bases institucionales previas que en muchos casos son legales -tratados y convenciones como la Declaración Universal de Derechos Humanos o la CMNUCC ${ }^{10}-\mathrm{u}$ organizacionales -Naciones Unidas o la omc, entre otras-.

Por último, en lo que respecta a la quinta categoría, si bien el LB y la DBe mencionan y definen temáticas, su mención no es repetitiva caso por caso como lo es en la $\mathrm{DBr}$-"seguridad", “energía”, "migración”, "drogas”, entre otros temas-. El LB y la DBe prefieren repetir simplemente la necesidad de abarcar múltiples áreas. También en el LB y en la DBe se aclara

\footnotetext{
9 A veces, el término "mutuo" se intercambia con el concepto “común”, pero en general, el LB y la DBe prefieren el término "mutuo" por tener una connotación menos uniforme que "común”.

10 Convención Marco de las Naciones Unidas sobre el Cambio Climático.
} 
que son solo áreas señaladas -por nombrar algunas- de entre otras también posibles que se podrían acordar. En este sentido, la DBe señala: "Estamos decididos a desarrollar diálogos en el marco de las áreas temáticas del Foro, entre otras adicionales acordadas por las partes".

Finalmente, queremos destacar la presencia de una alta frecuencia léxica en la DBe del término "China" y de los distintos órganos y funcionarios de gobierno. En cambio, en la $\mathrm{DBr}$ no es común la referencia a la UE o sus funcionarios. Consideramos que en el FCC esto se relaciona con una posición política y de promoción del liderazgo, como mencionamos en el punto uno de nuestra definición de estrategia. La DBr, por su parte, busca despolitizar su contenido institucional omitiendo actores. En este mismo sentido, por ejemplo, puede encontrarse una considerable frecuencia del término "política" en la DBe, pero referencias al término "políticas" en el caso de la DBr.

A continuación, presentamos un gráfico donde se agregan, en las cinco categorías, las frecuencias de los términos clave.

\section{Gráfico 4}

Frecuencias relativas de términos claves, por categorías, en la Declaración de Beijing, el Libro blanco y la Declaración de Bruselas

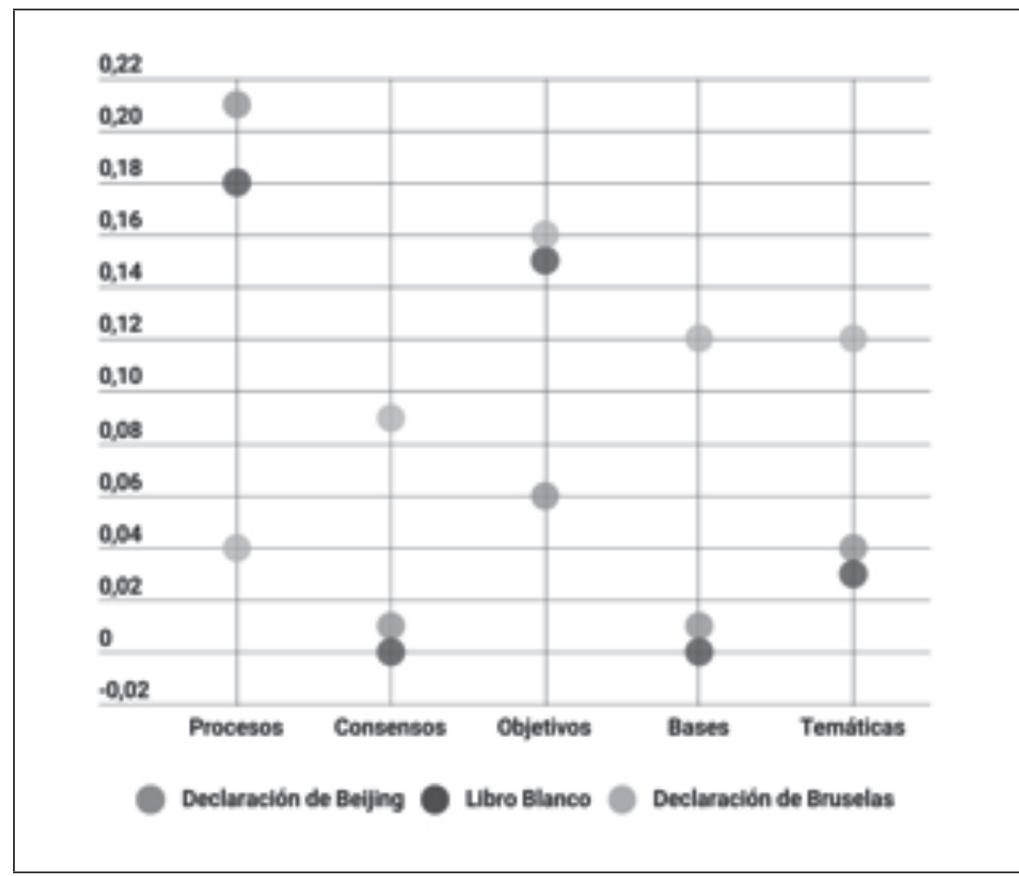

Fuente: elaboración propia con base en el Libro blanco hacia América Latina (2008), Declaración de Beijing (2015) y Declaración de Bruselas (2015). 
Como se puede observar, es claro el acercamiento entre el LB y la DBe en cuanto a las categorías analizadas, así como el distanciamiento de ambos documentos con respecto a la DBr. La única excepción parece ser la mención a objetivos, donde el LB y la DBr se acercan considerablemente. Esto se debe a que el LB, a diferencia de la $\mathrm{DBe}$, menciona de forma recurrente el objetivo del desarrollo. De todas formas, recordemos que la definición de objetivos busca ser mucho más concreta en la $\mathrm{DBr}$ que una referencia abierta al "desarrollo" como lo es en el LB. Además, distinto a lo que sucede en la $\mathrm{DBr}$, en el LB la frecuencia de procesos es mayor a la frecuencia de objetivos.

En general, el LB y la DBe utilizan el mismo lenguaje promoviendo la particularidad. Incluso, la DBe menciona en su texto que la propia declaración debe aplicarse según los principios de flexibilidad y de no condicionalidad. Estos dos principios son los ejes articuladores que permiten traducir la retórica discursiva china de la particularidad en aplicaciones institucionales. De esta forma, las instituciones particulares deben centrarse en procesos de diálogo y evitar bases rígidas externas-legales u organizacionales- por sobre los propios actores de la cooperación.

Finalmente, debemos resaltar que la $\mathrm{DBr}$, por su lado, fomenta bases institucionales formales, rígidas, estables y, en definitiva, uniformes y comunes para todos los países. Esto se produce con bases firmes en normas y temas preestablecidos, con objetivos y resultados ya determinados de antemano.

Así, esta se trata de una relevante diferencia entre el LB y la DBe, y por otra parte la DBr. Comprender la estrategia institucional de
China hacia América Latina implica considerar esta diferencia.

\section{b. Omnidireccionalidad}

La omnidireccionalidad es estudiada en este trabajo a partir de la promoción de múltiples direcciones temáticas presentes en el LB 2008 y en el Plan del FCC (2015-2019) y con fines comparativos en el Plan Celac-UE del 2015.

Una comparación de estos tres documentos indica que las temáticas de cooperación del Plan del FCC coinciden en un $88 \%$ con las direcciones temáticas del LB. Por su parte, en el caso del Plan Celac-UE, la coincidencia es del 35\% con el LB. En el anexo de este trabajo, pueden apreciarse las referencias a las relaciones por apartados de cada uno de estos tres documentos.

Nuevamente, es de destacar, que el LB y el Plan del fCC comparten un mismo lenguaje, siendo la relación entre direcciones temáticas una vinculación sin mayores diferencias de sentido. Las únicas excepciones que pueden mencionarse son referencias a temas como "gobernanza de internet”, "apoyo a las tecnologías de la información" y "fortalecimiento de pequeñas y medianas empresas", que se encuentran como temáticas en el Plan del FCC y ni siquiera se mencionan en el LB. El Plan del FCC omite temáticas como "condonación de deudas", "cooperación militar" y "seguridad no tradicional", que son temáticas establecidas en el LB.

Con respecto al Plan Celac-UE del año 2015, si bien hay coincidencia en varias direcciones temáticas con el LB y el Plan del FCC, lo cierto es que muchos de estos temas poseen enfoques diferentes o son valorizados de distinta manera en los documentos. Por ejemplo, 
la temática "energía" solo es referenciada en el Plan Celac-UE en el marco del enfoque del desarrollo sostenible -ahorro energético y energías renovables- y no como recurso económico, que es el enfoque dado en el LB y en el Plan del FCC. Asimismo, el "problema migratorio", la "lucha contra las drogas" y la "perspectiva de género", por ejemplo, tienen una preponderancia mucho mayor en el Plan Celac-UE que en el LB o en el Plan FCC. Luego, también, es muy claro lo que omite el Plan Celac-UE con respecto al LB y al Plan FCC. El Plan Celac-UE no refiere a la cooperación política -salvo cuando menciona marcos de apoyo en "asuntos internacionales"-, no refiere al comercio ni a sectores económicos, $\mathrm{y}$ tampoco a la cooperación cultural, que si son temáticas destacadas en el LB y en el Plan del FCC. En general, el Plan Celac-UE tiene un formato de menos temas -diez-, pero más detallados en cuanto a los resultados esperados.

A continuación, se presenta un diagrama con las coincidencias y diferencias más relevantes por direcciones temáticas, incluyendo las diferencias de sentido y de preponderancia ya mencionadas ${ }^{11}$.

\section{Diagrama 1}

\section{Direcciones temáticas y su intersección en el Plan del FCC y en el Plan Celac-UE}

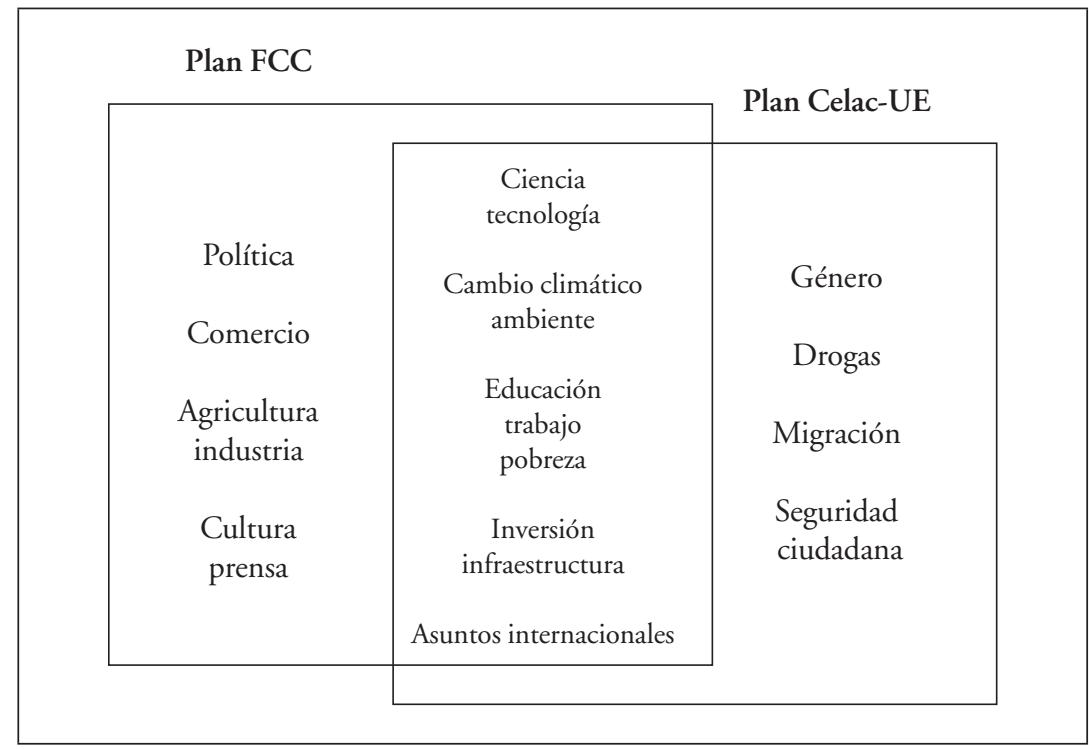

Fuente: elaboración propia con base en el Plan del FCC (2015-2019) y el Plan Celac-UE (2015).

11 Las direcciones temáticas en el diagrama se nombran con la terminología del LB, por ser la referencia comparativa utilizada. 
La cooperación política, la cooperación en comercio y por sectores económicos como la agricultura y la industria, así como la cooperación en cultura y prensa-por nombrar las más relevantes-, son temáticas de cooperación entre China y la Celac, pero no entre la Celac y la UE. Por su parte, temáticas como la cooperación en políticas con perspectiva de género, en el problema mundial de las drogas y en la seguridad ciudadana son destacadas de la Celac y la UE, pero no reciben el mismo tratamiento entre China y la Celac. En definitiva, la intersección muestra temas comunes de cooperación de la Celac tanto con China como con la UE. Los ejes de la ciencia y la tecnología; el cambio climático; la relación entre educación, trabajo y reducción de la pobreza; y la inversión en infraestructura se encuentran entre las más destacadas coincidencias. La cooperación en asuntos internacionales es la única base política de cooperación en la que coincide la Celac con China y con la UE.

Podemos afirmar que la sección política del Libro blanco y la Declaración de Beijing prefieren no mencionar las direcciones temáticas caso por caso. Cuando las temáticas se explicitan en la sección de cooperación omnidireccional del Libro blanco o en el Plan del FCC, la mención es extensa, pero no específica y solo con fines indicativos, como posibles áreas de cooperación. Por el contrario, en la Declaración de Bruselas y en el Plan CelacUE, las direcciones temáticas son listadas con minuciosa especificidad.

En este caso, es el principio de multiplicidad el eje articulador que traduce la estrategia discursiva china de la omnidireccionalidad en una práctica institucional. Se trata de un enfoque basado en la diversificación de áreas y temáticas, más que en la coordinación institucional. Además, las áreas y temáticas se mencionan siempre con fines indicativos y no exclusivos, para no contradecir los principios de flexibilidad y de no condicionalidad.

\section{CONCLUSIONES Y DISCUSIÓN}

A modo de conclusiones, presentamos cuatro puntos que comprueban nuestra hipótesis de trabajo.

En primer lugar, que el Libro blanco promueve la lógica de la particularidad, mientras que la Declaración de Beijing busca aplicar la particularidad institucionalmente bajo los principios de flexibilidad y de no condicionalidad. Por el contrario, y en segundo lugar, la Declaración de Bruselas es rígida, uniforme y prefiere reafirmar y aplicar bases legales u organizaciones preestablecidas. En tercer lugar, el Libro blanco fomenta la omnidireccionalidad, mientras que institucionalmente el Plan del FCC refiere a la multiplicidad de áreas y temáticas en el mismo sentido. Y por último, como cuarto punto, el Plan Celac-UE fomenta múltiples temáticas, aunque de forma más restringida y con mayor rigidez - debido a su especificidad- que el Libro blanco y el FCC. Además, en ejes de coincidencia, el Plan CelacUE posee enfoques distintos al Libro blanco y al Plan del FCc. De esta forma, decimos que el FCC aplica institucionalmente la estrategia del discurso del LB 2008 en sus dos dimensiones -fomento de la particularidad y promoción de la omnidireccionalidad-, mientras que la Celac no aplica estos patrones estratégicos con otras regiones. 
Como espacio de discusión, y en relación con nuestros hallazgos empíricos, mencionamos las siguientes cuestiones de interés.

La estrategia institucional de China hacia América Latina es canalizada por el Foro China-Celac y no por otras organizaciones de integración regional que tienen un volumen de interacción bajo con China. Esto, que fue comprobado empíricamente, se debe a condiciones de representación y a la posibilidad identificada por China para influir en la institucionalidad incipiente de la Celac. La composición de la Celac (con los treinta y tres Estados de América Latina y el Caribe) y su reciente conformación en diciembre de 2011 posibilitaron esta situación. En el marco del Foro China-Celac es donde China puede implementar institucionalmente su estrategia de fomento de la particularidad y de promoción de la omnidireccionalidad. Es decir, la posibilidad de influencia china-debido a su nueva posición estructural- queda materializada en reglas y prácticas institucionales explícitas en el marco del Foro. Esto fue comprobado empíricamente en la presente investigación y representa un desafío de entendimiento sobre la aplicación y el desarrollo futuro del Foro China-Celac.

La estrategia institucional de China hacia América Latina se caracteriza por la flexibilidad, la no condicionalidad y la multiplicidad. Esta forma institucional que adopta la estrategia china requiere que futuros análisis de tipo institucional, organizacional, de gestión y de estrategia sobre la relación entre China y América Latina -ya sea a nivel de país o de otro tipo de vinculaciones sectoriales-, consideren marcos conceptuales y metodológicos interdisciplinarios. Además, este tipo de es- trategia institucional representa un desafío de análisis constructivista - para futuras agendas de investigación de la relación entre China y América Latina- así como para enfoques estructuralistas.

Creemos, por un lado, que el estudio de las relaciones internacionales debe considerar una posible orientación en política exterior de la teoría constructivista, en particular con el caso de la diplomacia china y aunque esto contradiga la aplicación más frecuente de esta teoría.

Por otro lado, China y América Latina han crecido exponencialmente en sus vinculaciones económicas y políticas. El posicionamiento estructural de China y la falta de entendimiento sobre sus prácticas institucionales han posibilitado amplios debates en los últimos cuarenta años. Diversos estudios y autores han trabajado desde distintas disciplinas y enfoques la particularidad del modelo chino. Incluso las opiniones divergentes han tenido un importante impacto (positivo o negativo, según el punto de vista) en el propio crecimiento de las relaciones entre China y América Latina. Este trabajo es un aporte a esta discusión, para mejorar el entendimiento sobre China y, en particular, para interpretar la relación que se propone entre agente y estructura. La retórica y la institucionalidad china definen una específica forma para que los agentes (Estados) cooperen entre sí al mismo tiempo que ganan autonomía y escapan de los condicionamientos estructurales. La distancia entre esta propuesta china y la realidad en el marco de las condiciones materiales de la estructura internacional debe incentivar a nuevas investigaciones.

El FCC es un caso especial de aplicación de reglas identificables con estas característi- 
cas particulares. Por lo tanto, la preocupación institucional sobre el modelo chino de vinculación exterior pudo trabajarse empíricamente en la presente investigación. El trabajo realizado, consideramos, nos permite pensar en nuevas aplicaciones de la lógica cooperativa china y en las implementaciones directas e indirectas, formales e informales, de su estrategia institucional.

\section{REFERENCIAS}

Abdenur, A. y De Sousa Neto, D. (2013). La creciente influencia de China en el Atlántico Sur. Revista CIDOB d'Afers Internacionals, 102/103: 169-197.

Adler, E. (2005). Communitarian International Relations. The Epistemic Foundations of International Relations. Nueva York: Routledge.

Armony, A. y Pérez-Liñán, A. (2017). Introduction to the special issue - china and latin american political economy: How china became part of latin american studies. Issues and Studies, 53(1): 1-12.

Arrighi, G. (2007). Adam Smith en Pekin. Madrid: Akal. Arrighi, G. y Drangel, J. (1986). The stratification of the world-economy: an exploration of the semiperipheral zone. Review, Sage Publications, $\mathrm{X}(1)$ : 9-74.

Badía, J. F. (1973). Métodos en el estudio de la ciencia política. Revista Española de la Opinión Pública, Centro de Investigaciones Sociológicas, 31: 7-25.

Beeson, M. (2009). Hegemonic transition in East Asia? The dynamics of chinese and american power. Review of International Studies, Cambridge University Press, 35(1): 95-112.

Bolinaga, L. y Slipak, A. (2015). El Consenso de Beijing y la reprimarización productiva de América Latina: el caso argentino. Problemas del Desarrollo, UNAM, 46(183): 33-58.
Braun, E. y Suárez, E. (1973). China en las Naciones Unidas: una evaluación. Foro Internacional, El Colegio de México, XIII (3)51: 392-401.

Breault, D. y Callejo, D. (2012). Introduction to Case Studies. Counterpoints, 401: 59-62.

Casanueva, H. (2013). ALC-UE: ¿Construir juntos el futuro? Estudios Internacionales, Universidad de Chile, 45(176): 145-155.

Chaponnière, J. R. y Salama, P. (2016). L'Amerique Latine et la Chine: "Je t'aime... moi non plus". Revue d'Économie Financière, Association d'Economie Financiere, 124: 225-242.

Cheng, J. (2006). Latin America in China’s contemporary foreign policy. Journal of Contemporary Asia, Routledge, 36(4): 500-528.

Choo, J. (2009). China’s relations with Latin America: issues, policy, strategies, and implications. Journal of International and Area Studies, Seoul National University, 16(2): 71-90.

Chow, G. C. (2010). Interpreting China's Economy. Londres: World Scientific Publishing.

Cooper Ramo, J. (2004). The Beijing Consensus. Londres: The Foreign Policy Centre.

Corkin, L. (2014). China's Rising Soft Power: The Role of Rhetoric in constructing China-Africa relations. Revista Brasileira de Politica Internacional, 57: 49-72.

Cornejo, R. (2013). La relación de México con China, la política del desconcierto. Foro Internacional, El Colegio de México, 53(3-4): 645-666.

Creutzfeldt, B. (2013). América Latina en la política exterior china. Papel Politico, Pontificia Universidad Javeriana de Bogotá, 18(2): 599-611.

De Onis, J. (2013). Fractured continent: The turmoil and promise of Latin America. World Affairs, Sage Publications, Inc., 176(1): 35-42.

Ellis, E. (2009). China in Latin America: The Whats and Wherefores. Boulder: Lynne Rienner Publishers. 
Escudé, C. (2012). El realismo periférico y su relevancia teórica ante el ascenso de China. Desarrollo Económico, 51 (204): 529-542. http://www.jstor.org/ stable/23612357.

Fanjul, E. (2011). El "Consenso de Beijing": universalidad y particularidad del modelo chino. Información Comercial Española, ICE: Revista de Economía, Ministerio de Industria, Turismo y Comercio, 859: 47-53.

Finnemore, M. (1996). National Interests in International Society. New York: Cornell University Press.

Finnemore, M. y Sikkink, K. (2001). Taking Stock. The Constructivist Research Program in International Relations and Comparative Politics. Annual Review of Political Science, 4: 391-416.

Gálvez, L. (2012). China y los países en desarrollo: el caso de América Latina. Estudios Internacionales, Universidad de Chile, 171: 7-27.

Gao, H. (2007). Taming the Dragon: China's experience in the wто Dispute Settlement System. Legal Issues of Economic Integration, 34(4): 369-392.

Garner, R. E. (2008). Understanding Contemporary China. Boulder: Lynne Rienner.

George, A. y Bennett, A. (2005). Case Studies and Theory Development in the Social Sciences. Cambridge: The MIT Press.

Giddens, A. (2015). La constitución de la sociedad. Bases para la teoría de la estructuración. Buenos Aires: Amorrortu.

Gómez Díaz, D. A. (2017). El discurso confuciano en la política china. En Beltrán Antolín, J. (ed.), Viaje al centro. El XIX Congreso del Partido Comunista Chino (pp. 33-56). Barcelona: Edicions Bellaterra.

Greif, A. y Laitin, D. (2004). A theory of endogenous institutional change. American Political Science Review, American Political Science Association, 98(4): 633-652.
Hadfield, G. (2005). The Many Legal Institutions that Support Contractual Commitments. En Menard, C. y Shirley, M. (eds.), Handbook of New InstitutionalEconomics (pp. 175-204), New York: Springer.

Haro Navejas, F. y Hernández Mendoza, R. (2016). Viaje al oeste. La asociación sino-mexicana: una posibilidad funcionalista. Foro Internacional, El Colegio de México, 56(2): 409-449.

Haynes, K. (2017). Would China Be a Benign Hegemon? The Diplomat, 2 de junio de 2017. https:// thediplomat.com/2017/06/would-china-be-abenign-hegemon/

Herrera-Feligreras, A. (2017). En torno al socialismo con características chinas. En Beltrán Antolín, J. (ed.), Viaje al centro. El XIX Congreso del Partido Comunista Chino (pp. 77-94). Barcelona: Edicions Bellaterra.

Hofer, C. y Schendel, D. (1978). Strategy Formulation: Analytical Concepts. St. Paul: West Group.

Hopf, T. (1998). The Promise of Constructivism in International Relations Theory. International Security, The мIт Press, 23(1): 171-200.

Huang, Y. (2010). Debating China’s Economic Growth: The Beijing Consensus or The Washington Consensus. Academy of Management Perspectives, 24(2): 31-47.

Hunston, S. (1996). Evaluation and Organization in a Sample of Written Academic Discourse. En Coulthard, M. (ed.), Advances in Written Text Analysis (pp. 191-218). London: Routledge.

Ikenberry, J. (2008). The Rise of China and the Future of the West. Foreign Affairs, Council on Foreign Relations, 87(1): 23-37.

Jaguaribe, H. (1973). Dependencia y autonomía en América Latina. En Jaguaribe, H.; Ferrer, A.; Wionczek, M. S. y Dos Santos, T. (eds.), La dependencia político-económica de América Latina (pp. 1-85), México D. F.: Siglo Xxi Editores. 
Jaramillo, P.; Lehmann, S. y Moreno, D. (2009). China, precios de commodities y desempeño de América Latina: algunos hechos estilizados. Cuadernos de Economía, Pontificia Universidad Católica de Chile, 46(133): 67-105.

Jenkins, R. (2012). Latin America and China - a new dependency? Third World Quarterly, Routledge, 33(7): 1337-1358.

Kolbe, R. y Burnett, M. (1991). Content-Analysis Research: An Examination of Applications with Directives for Improving Research Reliability and Objectivity. Journal of Consumer Research, Oxford University Press, 18(2): 243-250.

Kotschwar, B. (2014). Chinas's economic influence in Latin America. Asian Economic Policy Review, Japan Center for Economic Research, 9(2): 202-222.

Leiteritz, R. y Coral, H. (2017). China como fuente de recursos financieros y de cooperación para América Latina y el Caribe: análisis comparativo con África. En Pastrana, E. y Gehring, H. (eds.), $L a$ proyección de China en América Latina y el Caribe (pp. 293-315). Bogotá: Pontificia Universidad Javeriana.

Li, C. (2014). The confucian philosophy of harmony. New York: Routledge.

Li, T. y Wu, Y. (2016). Pragmatism in China, Chinese Pragmatism. Paper presentado en la International Conference on Humanity, Education and Social Science (ICHess, 2016).

Lijphart, A. (1971). Comparative Politics and the Comparative Method. American Political Science Review, American Political Science Association, 65(3): 682-693.

Liu, J. (2017). Major-country diplomacy with Chinese characteristics reflects trends of the times. China International Studies, May/June 2017: 28-44.
López, J.; Rodil, O.; Martínez, G. y Valdez, S. (2015). The Emergence of China in Latin America. Artículo presentado en XIV International Business and Economy Conference 2015, Bangkok.

Lyons, J. (1995). Linguistic Semantics. An Introduction. Cambridge: Cambridge University Press.

McEnergy, A. y Wilson, A. (2001). Corpus Linguistics. Edinburgh: Edinburgh University Press.

Modelski, G. (1978). The long cycle of global politics and the nation-state. Comparative Studies in Society and History, 20(2): 214-235.

Mora, F. (1999). Sino-latin american relations: sources and consequences, 1977-1997. Journal of Interamerican Studies and World Affairs, University of Miami, 41(2): 91-116.

Mora, F. (1997). The People's Republic of China and Latin America: from indifference to engagement. Asian Affairs, Taylor \& Francis, Ltd., 24(1): 35-58.

Morales Ruvalcaba, D. (2017). El fin del ciclo hegemónico de Estados Unidos. Foreign Affairs Latinoamérica. http://revistafal.com/el-fin-del-ciclohegemonico-de-estados-unidos/

Morales Ruvalcaba, D. (2015). Poder, estructura y hegemonia: pautas para el estudio de la gobernanza internacional. Índice de Poder Mundial. Guadalajara.

Morales Ruvalcaba, D. (2013). Inside the BRIC: analysis of the semiperipheral character of Brazil, Russia, India and China. Austral, 2(4): 141-173.

North, D. (1993). Instituciones, cambio institucional y desempeño económico. México D.F.: Fondo de Cultura Económica.

Olson, M. (1993). Dictatorship, Democracy, and Development. American Political Science Review, 87(3): 567-576.

Pastrana, E. y Vera, D. (2017). Transición de poder y orden mundial: el ascenso global de China y su proyección. En Pastrana, E. y Gehring, H. (eds.), 
La proyección de China en América Latina y el Caribe (pp. 23-72). Bogotá: Pontificia Universidad Javeriana.

Peters, G. (1998). Comparative Politics: Theory and Methods. New York: New York University Press.

Porter, M. (2011). ¿Qué es la estrategia? Harvard Business Review, 89: 100-117.

Prime, P. (2006). China's wTo compliance. En HungGay, F.; Pei, C. y Zhang, K. (eds), China and the Challenge of Economic Globalization: The Impact of WTO membership (pp. 36-50). New York: M. E. Sharpe.

Ragin, C. (1987). The Comparative Method. Moving Beyond Qualitative and Quantitative Strategies. Berkeley: University of California Press.

Ray, R.; Gallagher, K.; López, A. y Sanborn, C. (2015). China in Latin America: Lessons for South-South Cooperation and Sustainable Development. Boston: Boston University - Global Economic Governance Initaitive.

Ríos Sierra, J. (2013). De la cumbre alc-UE de Madrid a la cumbre Celac-UE de Santiago de Chile. Expectativas de una asociación estratégica birregional. OASIS, Universidad Externado de Colombia, 18: 75-94.

Rocha Valencia, A. (2013). Desenvolvimiento regional y despliegue internacional de la Celac. En Preciado, J. (ed.), Anuario de la Integración Latinoamericana y Caribeña 2013 (pp. 153-158), Guadalajara: Universidad de Guadalajara/Fondo Editorial Universitario.

Rocha Valencia, A. (2014). La Celac en la cúspide de los procesos de integración regional: más allá de un foro político - diplomático y más acá de una organización política internacional. Contextualizaciones Latinoamericanas, Universidad de Guadalajara, 6(10): 1-10.
Rocha Valencia, A. y Morales Ruvalcaba, D. (2018). El poder nacional-internacional de los Estados. Una propuesta trans-estructural. Geopolitica(s), Universidad Complutense de Madrid, 9(1): 137-169.

Rodríguez, C.; Quiles, O. y Herrera, L. (2005). Teoría y práctica del análisis de datos cualitativos. Proceso general y criterios de calidad. Revista Internacional de Ciencias Sociales y Humanidades, Universidad Autónoma de Tamaulipas, XV(2): 133-154.

Rosales, O. (2015). Primer Foro de la Comunidad de Estados Latinoamericanos y Caribeños (Celac) y China. Explorando espacios de cooperación en comercio e Inversión. Santiago de Chile: Cepal.

Rosecrance, R. (1987). Long cycle theory and international relations. International organization, 41(2): 283-301.

Ruggie, J. (1998). What Makes the World Hang Together? Neo-Utilitarianism and the Social Constructivist Challenge. International Organization, The mIT Press, 52(4): 855-885.

Serbin, A. (2017). China y América Latina y el Caribe frente a un cambio de ciclo: narrativas y estrategias. En Pastrana, E. y Gehring, H. (eds.), La proyección de China en América Latina y el Caribe (pp. 73-97). Bogotá: Pontificia Universidad Javeriana/Fundación Konrad Adenauer.

Shixue, J. (2006). Recent development of sino-latin american relations and its implications. Estudios Internacionales, Universidad de Chile, 38(152): 19-41.

Sidel, M. (1983). Latin American Studies in the People's Republic of China. Latin American Research Review, The Latin American Studies Association, 18(1): 143-153

Sinclar, J. (1991). Corpus Concordance Collocation. Oxford: Oxford University Press.

$\mathrm{Su}, \mathrm{G}$. (2017). The great historic journey of Chinese diplomacy. China International Studies, November/ December: 5-39. 
Terlouw, K. (2002). The semiperipheral space in the world-system. Review, Fernand Braudel Center, 25(1): 1-22.

Tokatlian, J. G. y Carvajal, L. (1995). Autonomía y política exterior: un debate abierto, un futuro incierto. Revista СIDOB d'Afers Internacionals, Barcelona Centre for International Affairs, 28: 7-31.

Tzili, E. (2017). Antecedentes y proyecciones del Foro China-Celac y su influencia en la dinámica hemisférica. En Pastrana, E. y Gehring, H. (eds.), La proyección de China en América Latina y el Caribe (pp. 145-169). Bogotá: Pontificia Universidad Javeriana/Fundación Konrad Adenauer.

Urdinez, F. y Maseiro, G. (2015). China and the wто: Will the Market Economy Status Make Any Difference after 2016? Chinese Economy, 48(2): 155-172.

Vitelli, M. (2014). Veinte años de constructivismo en Relaciones Internacionales. Del debate metateórico al desarrollo de investigaciones empíricas. Una perspectiva sin un marco de política exterior. POSTData, 19(1): 129-162.

Walker, I. y Casanueva, H. (2012). Hacia una sociedad de actores globales: Las relaciones entre Europa y América Latina en el nuevo contexto global. Estudios Internacionales, Universidad de Chile, 44(172): 101-111.
Wallerstein, I. (2003a). El moderno sistema mundial. Tomo I: La agricultura capitalista y los orígenes de la economía-mundo europea en el siglo XVI. México D.F.: Siglo XxI.

Wallerstein, I. (2003b). El moderno sistema mundial. Tomo II: El mercantilismo y la consolidación de la economía-mundo europea 1600-1750. México D. F.: Siglo XXI.

Wang, H. (1988). Changing Chinese Political Cultural Structure. Journal of Fudan University, 3: 55-64.

Wendt, A. (1999). Social Theory of International Politics. New York: Cambridge University Press.

Xi, J. (2014). La gobernación y administración de China. Beijing: Ediciones en Lenguas Extranjeras Cía. Ltda.

Yongtao, L. (2012). Promote china-latin american relations in the 21st Century. Revista De Globalización, Competitividad y Gobernabilidad, 6(1): 107-114.

Yu, L. (2015). China's strategic partnership with Latin America: A fulcrum in China's rise. International Affairs, The Royal Institute of International Affairs, 91(5): 1047-1068.

Zhang, S.-G. y Wang, N.-K. (1988). Latin American Studies in the People's Republic of China: Current and Future Prospects. Latin American Research Review, The Latin American Studies Association, 23(1): 123-132. 
ANEXO

Tabla 1

Direcciones temáticas del Libro blanco y relaciones con apartados del Plan del FCC y el Plan Celac-UE

\begin{tabular}{|c|c|c|}
\hline Dirección LB* & Plan FCC & Plan Celac-UE \\
\hline 1po. Intercambio de alto nivel & II & \\
\hline 2po. Intercambio entre los órganos legislativos & $\mathrm{I} 2$ & \\
\hline 3po. Intercambio entre los partidos políticos & $\mathrm{I}_{3}$ & \\
\hline 4po. Mecanismos de consulta & II & \\
\hline 5po. Cooperación en asuntos internacionales & $\mathrm{III}_{1} \mathrm{II}_{3}$ & $2,3,8$ \\
\hline 6po. Contactos entre los gobiernos locales & $\mathrm{I}_{3}$ & \\
\hline 1e. Comercio & IIII, III2 & \\
\hline 2e. Cooperación en inversión & $\mathrm{IIII}_{\mathrm{I}} \mathrm{III}_{3}, \mathrm{IV}_{3}$ & 8 \\
\hline 3e. Cooperación financiera & $\mathrm{III}_{7}$, III8 & \\
\hline 4e. Cooperación agrícola & VII, $\mathrm{VI}_{2}, \mathrm{VI}_{3}, \mathrm{VI}_{4}, \mathrm{VI}_{5}$ & \\
\hline 5e. Cooperación industrial & $\mathrm{V}_{5}, \mathrm{VIII}_{1}, \mathrm{VII}_{2}$ & \\
\hline 6e. Construcción de infraestructuras & IVI, IV $2, V_{3}$ & 3 \\
\hline 7e. Cooperación en recursos y energías & $V_{1}, V_{2}, V_{3}, V_{4}$ & \\
\hline 8e. Cooperación aduanera & III6 & \\
\hline 9e. Cooperación en inspección de calidad y cuarentena & III6 & \\
\hline 10e. Cooperación turística & $\mathrm{XI}, \mathrm{XI}_{2}, \mathrm{XI}_{3}, \mathrm{XI}_{4}$ & \\
\hline \multicolumn{3}{|l|}{ 11e. Reducción y condonación de deudas } \\
\hline \multicolumn{3}{|l|}{ 12e. Asistencia económica y técnica } \\
\hline 13e. Cooperación multilateral & $\mathrm{II}_{2}$ & \\
\hline 14e. Cooperación entre las cámaras y promotoras de comercio & $\mathrm{III}_{4}$ & \\
\hline 1c. Intercambio cultural y deportivo & $\mathrm{IX}_{\mathrm{I}}, \mathrm{IX}_{2}, \mathrm{IX}_{4}, \mathrm{X}_{3}$ & \\
\hline 2c. Cooperación científica, tecnológica y educativa & $\begin{array}{l}\mathrm{VII}_{2}, \mathrm{VII}_{4}, \mathrm{VII}_{5}, \mathrm{VII} 6, \mathrm{VII}_{7} \text {, } \\
\mathrm{VIII}_{2}\end{array}$ & $1,2,9$ \\
\hline 3c. Cooperación médica y sanitaria & XII6 & \\
\hline 4c. Cooperación consular e intercambio de personal & $\mathrm{I}_{4}$ & 4 \\
\hline 5c. Cooperación en prensa & $\mathrm{XI}_{1}, \mathrm{X}_{2}$ & \\
\hline 6c. Intercambio entre los pueblos & VIII, VIII, XIIII, XIII2 & 7,9 \\
\hline 7c. Cooperación en la protección del medio ambiente & $\mathrm{XII}_{2}$ & 2 \\
\hline 8c. Cooperación contra el cambio climático & $\mathrm{II}_{4}, \mathrm{XIII}$ & 2 \\
\hline 9c. Cooperación en recursos humanos y seguridad social & VIII2, VIII6 & 3,5 \\
\hline
\end{tabular}




\begin{tabular}{|l|l|l|}
\hline Dirección $\mathrm{LB}^{*}$ & PLAN FCC & Plan Celac-UE \\
\hline $\begin{array}{l}\text { 10c. Reducción de calamidades, socorro a damnificados y asis- } \\
\text { tencia humanitaria }\end{array}$ & $\mathrm{XII}_{3}, \mathrm{XII}_{4}$ & \\
\hline 11c. Cooperación en el alivio de la pobreza & $\mathrm{VI}_{6}, \mathrm{XII}_{5}$ & 3 \\
\hline 1pa. Intercambio y colaboración militares & & 6 \\
\hline 2pa. Cooperación judicial y policial & $\mathrm{I}_{5}, \mathrm{I} 6$ & 6 \\
\hline 3pa. Seguridad no tradicional & & 10 \\
\hline
\end{tabular}

Fuente: elaboración propia con base en el Documento sobre la Política de China hacia América Latina y el Caribe (2008), el Plan Foro China-Celac (2015-2019) y el Plan Celac-UE (2015).

* Las direcciones temáticas poseen una numeración dentro de cada área de cooperación del Libro blanco: Política (po) del 1 al 6; Económica (e) del 1 al 14; Cultural y Social (c) del 1 al 11; Paz, Seguridad y Justicia (pa) del 1 al 3. 